\title{
Elaboração e Catalogação de Atividades com Objetos de Aprendizagem de Física no Repositório de Conteúdos Digitais InterRed
}

\section{(Preparation and Cataloging of Activities involving Physics Learning Objects in the InterRed, a Repository of Digital Content)}

\author{
Maurício Dourado - Universidade Federal do Ceará \\ cmauriciojd@gmail.com \\ Gilvandenys Leite Sales - Centro Federal de Educação Tecnológica do Ceará \\ denyssales@cefetce.br \\ Giovanni Cordeiro Barroso - Universidade Federal do Ceará \\ gcb@fisica.ufc.br \\ José Marques Soares - Centro Federal de Educação Tecnológica do Ceará \\ marques@cefetce.br \\ Eliana Moreira de Oliveira - Centro Federal de Educação Tecnológica do Ceará \\ eaufcmidias@gmail.com \\ Paula Patrícia Barbosa Ventura - Universidade Federal do Ceará \\ paula@virtual.ufc.br
}

Resumo. A crescente produção de Objetos de Aprendizagem motivou a criação de repositórios para sua catalogação na rede web. Neste trabalho apresenta-se uma metodologia de desenvolvimento de atividades para alimentar o repositório de conteúdos digitais denominado InterRed, coordenado pelo Centro Federal de Educação Tecnológica do Ceará (CEFETCE). A metodologia que se propõe é fruto de discussões do curso de Licenciatura em Física do CEFET-CE. Construída a atividade, têm-se conteúdos digitais prontos para dar suporte ao professor e possibilitar uma aprendizagem mais significativa ao aprendiz.

Palavras-chave: Objetos de Aprendizagem, InterRed, Atividades de Física.

Abstract. The increasing production of learning objects has led to the creation of cataloging his archives for the network web. In this paper presents a methodology for development of activities to feed the repository of digital content called InterRed, coordinated by the Federal Technological Education Center of Ceará (CEFET-CE). The methodology that is proposed is the result of discussions within the course of Degree in Physics from CEFET-CE. Built the activity, have digital content ready to support the teacher and allow a more significant learning to learner.

Keywords: Learning Objects, InterRed, Activities of Physics. 


\section{Tecnologias Digitais, Formação e Capacitação Permanente de Professores}

A crescente utilização das tecnologias digitais na esfera educacional tem proporcionado benefícios à formação docente. No entanto, é preciso que os professores saibam utilizar os recursos tecnológicos em favor da aprendizagem dos alunos. Para que isso seja possível, é imprescindível investir em sua formação. Nunes (2007) argumenta que os professores já tem consciência da necessidade desse processo formativo, mas que o uso efetivo das tecnologias digitais em sala de aula ainda é preocupante.

Para apoio e suporte ao professor da educação básica, o governo disponibiliza um site que oferece ferramentas que auxilia diretamente o professor em sua práxis pedagógica (Portal do Professor, 2008), bem como oferece ao docente da educação profissional e tecnológica um ambiente de consultas e download de conteúdos pedagógicos digitais para aplicação direta na sala de aula (InterRed, 2008). Nestes portais, disponibilizam-se objetos de aprendizagem para downloads gratuitos na rede web.

Castro Filho (2007) enfatiza que as discussões sobre tecnologia e educação suscitam questões sobre o papel do professor, uma vez que os avanços tecnológicos são incorporados as práticas educacionais e que os problemas quanto ao uso da tecnologia advêm não só da falta de formação, mas também do acesso dos professores a essa tecnologia que chega à escola.

Ao mesmo tempo em que se faz necessário formar profissionais para atuar em cursos de formação, deve-se valorizar a capacidade de inovar, atuar e (re) criar conteúdos, o que requer também uma formação mais ampla e não apenas uma formação específica, o que antes correspondia somente a uma formação específica em sua área do conhecimento. Limitado à área de ciências (Física e Química especificamente), se exigia uma formação de conhecimentos práticos, técnicos e de resolução imediata, o que foi preciso repensar uma formação que ultrapassasse os conhecimentos do cotidiano, levando os alunos a serem autônomos e construtores de seu próprio conhecimento.

Diante do novo cenário educacional, questiona-se: qual o papel do professor frente ao uso das tecnologias digitais? Que tecnologias o professor de ciências pode utilizar em favor da aprendizagem dos alunos? Tais questionamentos vão de encontro com os Parâmetros Curriculares Nacionais (2002) que afirmam não ser possível "em pleno século 21, abrir mão dos recursos oferecidos pela tecnologia da informação e da comunicação e da capacitação dos professores para a utilização plena desses recursos", este documento estabelece também que “(...) é inadiável nosso esforço em mudar atitudes refratárias a seu uso, uma vez que estão amplamente disseminados na vida social em geral".

Este trabalho apresenta uma metodologia de construção de atividades com objetos de aprendizagem na área da Física a ser disponibilizada na rede web, por meio do repositório InterRed, como meio de auxiliar à formação inicial do professor, e servir de suporte a sua formação continuada.

$\mathrm{Na}$ próxima seção discutir-se-á acerca do repositório InterRed, na seção 3 aborda-se a metodologia de construção de atividades envolvendo conteúdos digitais dispostos na web, na seção 4 faz-se uma análise dos resultados dessa pesquisa e na seção 5, tecem-se considerações finais. 


\section{O Repositório de Objetos de Aprendizagem InterRed}

A expansão da Educação Profissional e Tecnológica (EPT) representa uma da metas do Governo Federal e vem sendo efetivada por meio de investimentos na expansão da Rede Federal de Educação Tecnológica. Nesse contexto, diversas instituições da Rede Federal de Ensino Profissional e Tecnológico estão provendo uma cultura de compartilhamento e trabalho colaborativo, visando o aumento quantitativo e qualitativo da oferta de recursos de apoio à EPT.

Nesta perspectiva, deu-se a concepção do InterRed (Figura 1) (InterRed, 2008) um sistema com foco no compartilhamento de conteúdos pedagógicos e metodologias, em que se incentivam a constante troca de experiências e aprendizado mútuo entre os profissionais vinculados à EPT de todo o país.

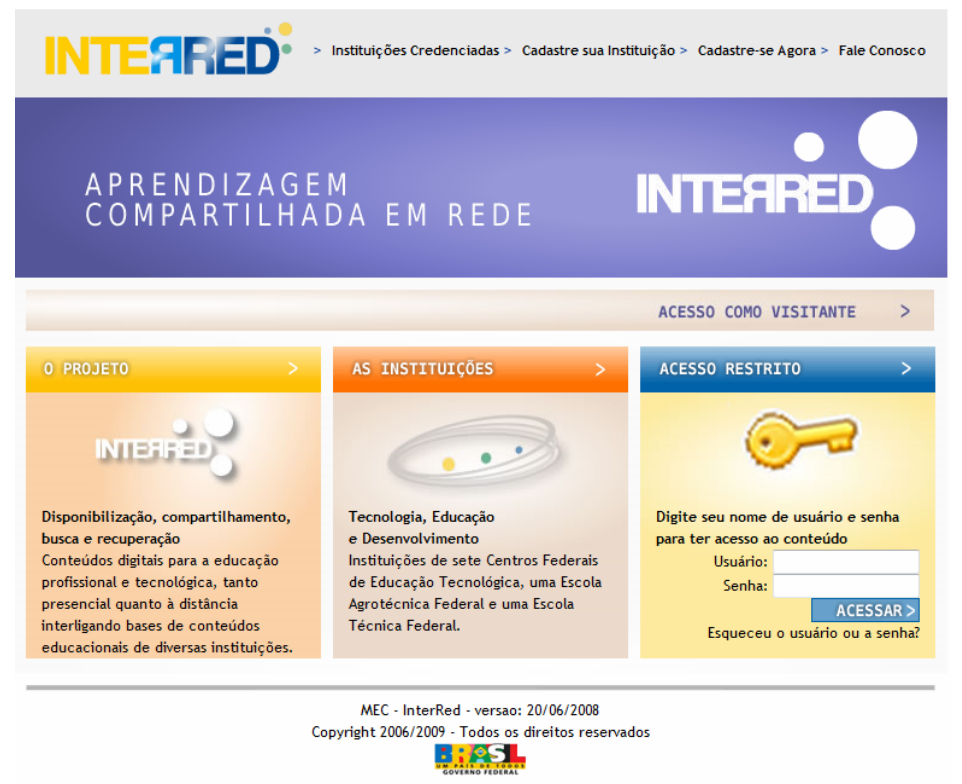

Figura 1 - Tela de abertura do InterRed

$\mathrm{Na}$ dinâmica da construção colaborativa, quanto mais intensa a participação e a interatividade entre os membros do projeto, maior o desenvolvimento do conhecimento. O principal produto desse projeto é a colaboração entre as partes e o amadurecimento de práticas interinstitucionais, criando possibilidades de apoio a profissionais e alunos, promovendo maior integração e interatividade na construção de conhecimentos em diversos domínios. Como o foco é a participação, evidencia-se a necessidade de uma retro-alimentação permanente de conteúdos e de conhecimentos, incentivando o acesso freqüente ao ambiente em busca de novidade.

$\mathrm{O}$ ambiente InterRed prevê uma política de compartilhamento em que os consumidores dos conteúdos são também provedores dos mesmos. No sistema inseremse seis perfis de usuários: Autor com acesso irrestrito ao repositório do sistema, responsável por alimentá-lo e publicar seus objetos de aprendizagem, bem como mantêlos atualizados; o Administrador, responsável pela manutenção geral do sistema e pela aprovação do credenciamento de novas instituições; o Coordenador, responsável pela administração no contexto de sua própria instituição; o Designado associado a um autor, responsável em catalogar objetos em nome do mesmo; o Catalogador que tem função 
semelhante a do designado, catalogar objetos que ficarão sob responsabilidade do autor, mas diferentemente do designado, o catalogador tem uma função institucional.

Com o objetivo de socializar o acesso ao InterRed, elenca-se ainda o perfil de Visitante, como aquele usuário que pode acessar e fazer download de todo o conteúdo disponibilizado, porém não é permitido que ele faça catalogação de objetos de aprendizagem (OA) de sua autoria no ambiente.

Um OA pode ser compreendido como qualquer entidade, digital ou não, que pode ser usada, reutilizada ou referenciada durante o processo de aprendizagem provido por algum tipo de tecnologia (IEEE, 2008). O significado de OA pode ser compreendido também sob o ponto de vista do paradigma da orientação a objetos. Wiley (2002) lembra que, como um objeto convencional dentro do citado paradigma de programação, um OA pode ser reusado em múltiplos contextos.

Nesta perspectiva, nota-se que um OA deve guardar uma característica granular e bem encapsulada, definindo sua própria interface pública. Sendo um fragmento, um OA, sozinho ou associado a outros, compõe um conteúdo aplicável ao processo de ensino e aprendizagem.

Muitos professores utilizam programas de computador como ferramentas de apoio à construção de material instrucional, mas raramente utilizam algum tipo de padrão. Entende-se ser necessário o desenvolvimento de uma cultura de compartilhamento interinstitucional, passando por um processo natural de aprendizado e de aprovação da comunidade.

Pelo exposto, o conceito fragmentar de OA no InterRed foi relaxado quanto à granularidade, a fim de evitar um provável esvaziamento do repositório. Dessa maneira, permite-se a catalogação de pequenos grãos como imagens, gráficos ou tabelas, mas também documentos inteiros e complexos, desde que se possam identificar os objetivos educacionais aos quais estes se destinam.

No dia 23 de julho de 2008, o InterRed, inicialmente restrito ao acesso de parceiros, foi liberado para visitantes. A restrição de visibilidade a um conteúdo é de responsabilidade de seu catalogador, que deverá indicar explicitamente se o acesso ao conteúdo é público ou privado. A fim de acompanhar o volume de acesso e o desenvolvimento do interesse da comunidade, o InterRed é monitorado pela ferramenta Google Analytics (http://www.google.com/analytics), que registra, entre outros aspectos, a quantidade de acessos por localidade, o tempo de permanência, índice de rejeição, lealdade do visitante, links mais acessados e até mesmo informação sobre o tipo de navegador e sistema operacional do usuário.

Este repositório representa uma ferramenta, não só como recurso para o apoio ao processo de ensino e aprendizagem, mas como elemento catalisador de experiências, aglutinando ao seu redor uma comunidade de interesses comuns. O percurso de desenvolvimento do InterRed, possui características de colaboração e aprendizagem coletiva, seja para o desenvolvimento de aplicações computacionais ou na elaboração e o compartilhamento dos conteúdos.

$\mathrm{Na}$ seção a seguir apresenta-se uma proposta de elaboração de atividades para alimentar o repositório InterRed.

\section{Metodologia para Elaboração de Atividades de Física}

Durante o primeiro semestre de 2008, na disciplina de metodologia do ensino de Física, os alunos do curso de Licenciatura de Física do CEFETCE fizeram uma pesquisa na 
rede web em busca de applets, simulações e software de Física, bem como objetos de aprendizagem de Física da Rede Interativa Virtual de Aprendizagem (RIVED), Laboratório Virtual de Física da PUC (LabVirt), projeto CESTA-UFRGS e o próprio InterRed.

Após a busca dos recursos digitais de Física na rede web os alunos sentiram a necessidade de elaborar atividades para serem aplicadas diretamente em sala de aula com esses recursos, bem como catalogá-los e usar o repositório do InterRed para este fim. Em seguida, as discussões versaram sobre a proposta de elaboração das atividades, sendo composta por quatro questionamentos acerca do tópico a ser explorado, a saber: O que se pretende? Onde encontrar o OA? Como utilizar o OA? Qual sua tarefa?

Dentro do quesito, "O que se pretende?" relatam-se os objetivos acerca do que se deseja que os alunos aprendam com a atividade digital. Para o quesito, "Onde encontrar o OA?" fornece-se ao usuário o endereço disponível do OA na web. Para o questionamento, "Como utilizar o OA?", é feita uma explanação dos procedimentos que se deve fazer para a utilização do OA. E ainda, para a pergunta, "Qual sua tarefa?" aplicam-se situações-problema para o aluno referente aos conteúdos abordados no OA.

\subsection{Desenvolvendo Atividades}

Dentre as atividades desenvolvidas foram abordados os tópicos da radiação do corpo negro, espectro eletromagnético, efeito fotoelétrico, produção e consumo de energia elétrica e estudo do movimento.

Apresenta-se a seguir (Figura 2) uma das atividades elaboradas para alimentar o InterRed em consonância com essa proposta.

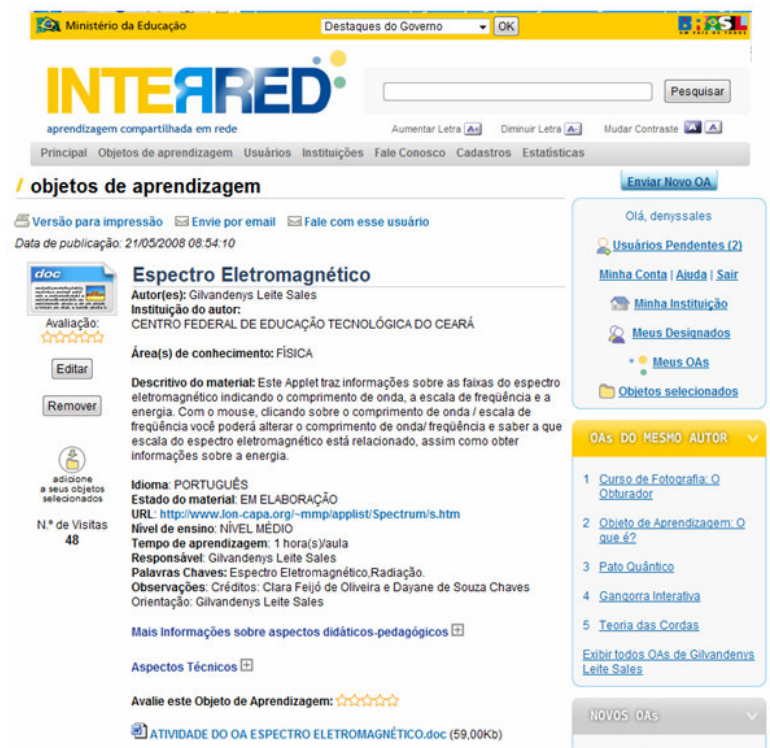

Figura 2 - Atividade com o OA Espectro Eletromagnético

A Atividade com o objeto de aprendizagem (OA) Espectro Eletromagnético ficou assim determinada:

\section{O que se pretende:}


-Compreender o espectro eletromagnético;

- Associar a Lei de Deslocamento de Wien com o espectro eletromagnético.

\section{Onde encontrar o OA:}

-Vá ao endereço

http://www.lon-capa.org/ mmp/applist/Spectrum/s.htm. Agora é só explorá-lo.

\section{Como utilizar o OA:}

-Este Applet (Figura 3) traz informações sobre as faixas do espectro eletromagnético indicando o comprimento de onda, a escala de freqüência e a energia. Com o mouse, clicando sobre o comprimento de onda/escala de freqüência você poderá alterar o comprimento de onda/ freqüência e saber a que escala do espectro eletromagnético está relacionado, assim como obter informações sobre a energia e aplicações reais daquela faixa de freqüência.

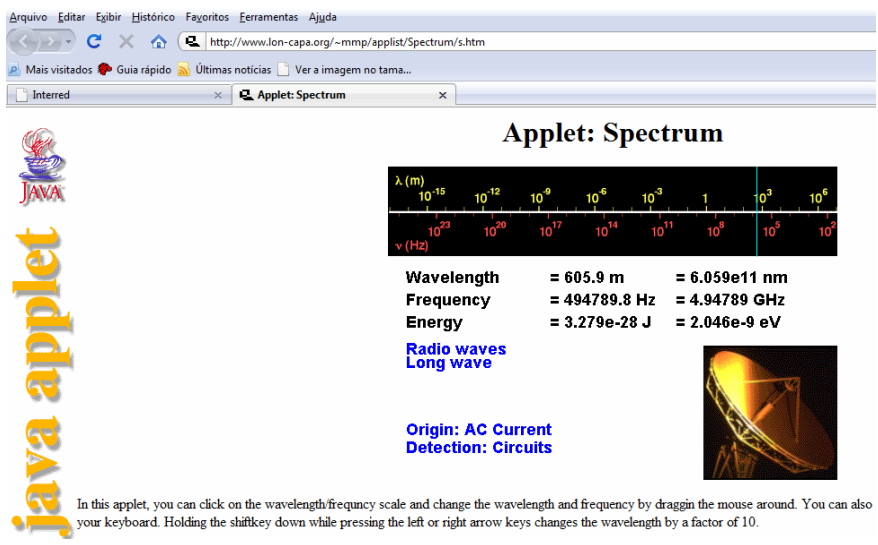

Figura 3 - Applet Spectrum

\section{Qual a sua tarefa?}

1 - Você deverá descobrir a relação da Lei de Deslocamento de Wien com as faixas do espectro eletromagnético.

Procedimentos:

- Utilize a Lei do Deslocamento de Wien para descobrir o comprimento de onda;

- Associe o comprimento de onda com a faixa do espectro eletromagnético.

Para as temperaturas (Quadro 1) obtenha qual a faixa do espectro eletromagnético.

\begin{tabular}{|c|c|}
\hline Temperatura & Faixa do espectro eletromagnético \\
\hline $9,4.10^{6} \mathrm{~K}$ & \\
\hline $1200 \mathrm{~K}$ & \\
\hline $0,29 \mathrm{~K}$ & \\
\hline $3,8.10^{3} \mathrm{~K}$ & \\
\hline
\end{tabular}

Quadro 1 - Relação Temperatura-Freqüência 
2- A radiação ultravioleta é emitida em grande parte pelo sol em direção ao nosso planeta, sendo esta absorvida pela camada de ozônio, protegendo-nos dessa perigosa radiação eletromagnética, que dependendo da quantidade de radiação ultravioleta que somos sujeitos, podem causar danos à saúde como o câncer de pele. Explorando o Applet Spectrum, aproximadamente em que faixas de frequiência encontram-se esses raios ultravioletas?

3- Os Raios X, descobertos por Röntegen em 1895, têm propriedade de atravessar matérias de baixa densidade, como nossos músculos, e de serem absorvidos por materiais de densidade mais elevada, como os ossos do nosso corpo. Quais são aproximadamente o mínimo e o máximo da energia desses raios?

Uma vez desenvolvida e debatida entre os membros da equipe a atividade, passa-se a fase seguinte que é a sua alocação no repositório InterRed, que é tratado na seção a seguir.

\subsection{Catalogando Atividades}

Construída a atividade, que deve estar disponível na web de forma a permitir alterações de quem quer que a acesse, têm-se conteúdos digitais prontos para dar suporte ao professor e possibilitar uma aprendizagem mais significativa ao aprendiz.

O procedimento de publicação no InterRed consiste basicamente em preencher os metadados previstos na ficha de catalogação (Figura 4).

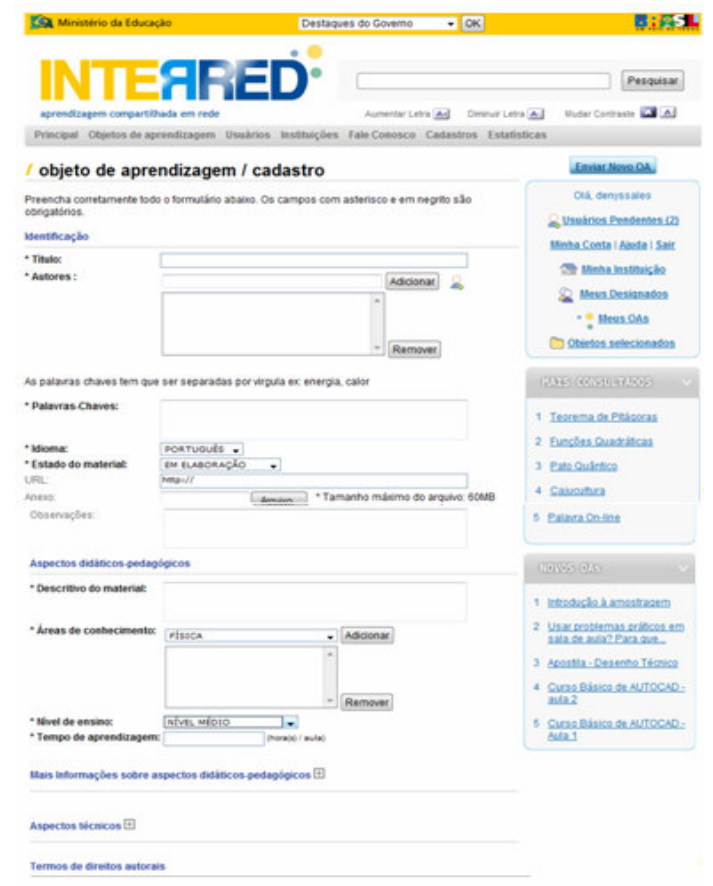

Figura 4 - Cadastramento de OA no InterRed

Além de contribuir para alimentar o repositório InterRed, tal procedimento visa facilitar o uso desses conteúdos digitais por parte do professor na sua sala de aula.

\section{Resultados}

O ambiente InterRed fornece estatísticas de acesso (Figura 5). 


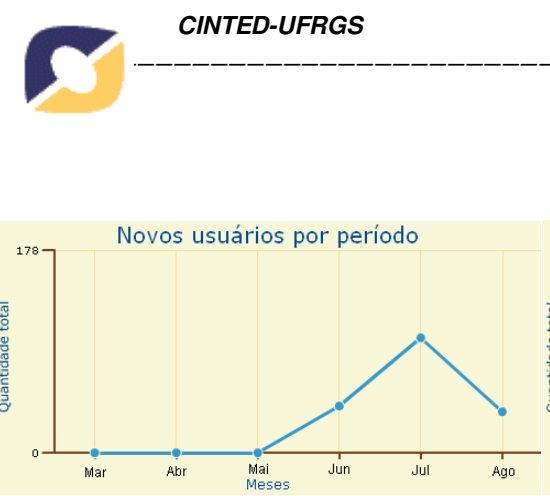

(a)

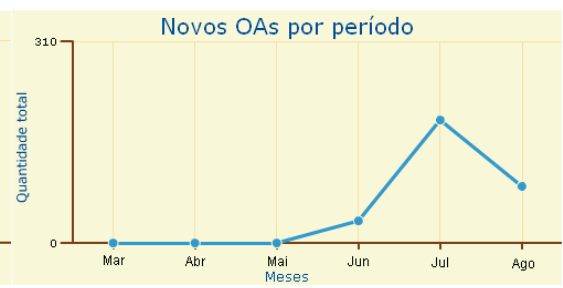

(b)

Figura 5. (a) Novos usuários/mês; (b) Novos OAs/mês; (c) OAs por Instituição

Relativo aos "Novos usuários por período" (Figura 5-a) percebe-se que houve um acentuado crescimento de acessos no mês de julho, uma vez que foi este o período em que o ambiente foi aberto aos visitantes.

Analisando o item "Novos OAs por período" (Figura 5-b) vê-se que a contribuição foi relevante, pois há um pico no mês de julho, ocasião em que os alunos da Licenciatura estavam apresentando seus resultados na disciplina de Metodologia do Ensino e alimentando o repositório InterRed. Tal fato contribuiu também para que a instituição, CEFET-CE, tornasse a instituição com maior número de OAs (Figura 5-c).

Na tela principal de abertura do InterRed (Figura 6) a contribuição da Instituição citada já garante um dos mais acessados OAs, o Pato Quântico, que consiste em uma atividade sobre o efeito fotoelétrico.

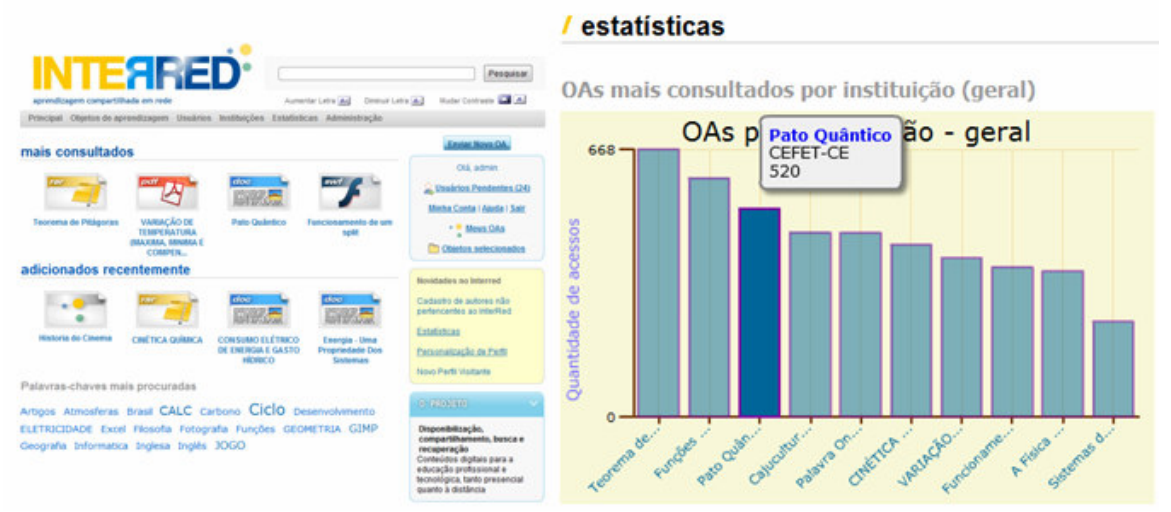

Figura 6 - OA mais consultado no InterRed

\section{Conclusões}

Considerando a problemática apresentada no que concerne à formação de professores no ensino de Ciências (especificamente na área de Física), se valida à questão de se privilegiar a tarefa de capacitar docentes nessa área tendo como pressuposto a inovação na práxis educativa e uma formação que ultrapasse a visão conservadora de ensinar Física, cuja ação docente se resume, intencionalmente, em repassar fórmulas acerca dos conteúdos a serem ensinados. Só a transmissão de informações e a destreza de habilidades operacionais, não são suficientes para a construção de conhecimentos.

Assim, a proposta metodológica de elaboração de atividades com objetos de aprendizagem de Física e catalogação em repositórios de conteúdos digitais, em que se utilizou o InterRed serviu como uma ferramenta a mais ao trabalho do professor, em 
que se aplicou não apenas uma metodologia que incitasse as trocas de experiências, mas a necessidade de elaborar atividades a serem desenvolvidas em sala de aula, o que pode levar o professor em serviço a repensar a sua prática pedagógica.

Espera-se que atividades elaboradas e depositadas no repositório de objetos de aprendizagem InterRed possam vir a ser um instrumento capaz de auxiliar a aprendizagem coletiva e compartilhada, levando alunos e professores a discussões e reflexões acerca dos conteúdos disponibilizados, desta forma contribuindo para uma aprendizagem significativa de Física.

\section{Referências Bibliográficas}

APPLET SPECTRUM. [online]. Disponível em: <http://www.loncapa.org/ mmp/applist/Spectrum/s.htm>. Acesso em: Julho de 2008.

BRASIL. Ministério da Educação e do Desporto. PCNs+ Ensino Médio: orientações educacionais complementares aos Parâmetros Curriculares Nacionais. Ciências da Natureza, Matemática e suas Tecnologias. Brasília, 2002.

CASTRO FILHO, J. A. de. Tecnologia, educação e formação de professores: superando dificuldades históricas. In: Sales, José Albino Moreira de. et al (orgs.). Formação e práticas docentes. Fortaleza: EdUECE, 2007.

IEEE LTSC - IEEE Learning Technology Standards Committee. [online]. Disponível em <http://ieeeltsc.org/ >. Acesso em: Agosto de 2008.

InterRed. Disponível em: <http://interred.cefetce.br>; Acesso em: 04 ago 2008.

Nunes, João B. C. Formação de professores para a sociedade do conhecimento: autonomia, colaboração e tecnologias livres. In: Sales, José Albino Moreira de. et al (orgs.). Formação e práticas docentes. Fortaleza: EdUECE, 2007.

Portal do Professor. [online]. Disponível em: < http://portaldoprofessor.mec.gov.br> Acesso em: 01 ago 2008.

RIVED - Rede Interativa Virtual de Educação. [online]. Disponível em: <http://www.rived.mec.gov.br> Acesso em: 01 ago 2008.

WILEY, D. A. The Instructional Use of Learning Objects. Agency for Instructional Technology. ISBN: 0-7842-0892-1. 1.ed. Janeiro, 2002. 